\title{
Filigrane
}

Écoutes psychanalytiques

\section{Transmission de la capacité négative : de la formation à la rencontre clinique}

\section{Réal Laperrière}

Volume 27, numéro 1, 2018

La transmission de la psychanalyse

URI : https://id.erudit.org/iderudit/1055602ar

DOI : https://doi.org/10.7202/1055602ar

Aller au sommaire du numéro

\section{Éditeur(s)}

Revue Santé mentale au Québec

ISSN

1192-1412 (imprimé)

1911-4656 (numérique)

Découvrir la revue

\section{Citer cet article}

Laperrière, R. (2018). Transmission de la capacité négative : de la formation à la rencontre clinique. Filigrane, 27(1), 45-60.

https://doi.org/10.7202/1055602ar

\section{Résumé de l'article}

Inspiré par le poète anglais John Keats, W. R. Bion considérait la capacité négative comme la qualité principale du psychanalyste, et il en faisait aussi pour le patient l'un des buts du traitement analytique. Cette capacité négative peut être mise en lien avec des concepts élaborés par différents auteurs, dont Freud (le masochisme érogène), Rosenberg (le masochisme gardien de la vie), De M'Uzan (l'inquiétude permanente), Press (la construction d'une position passive), Green (la passivité et la passivation), Ribas (la passivité de vie) et Phillips (être un embarras, être perdu, être impuissant). Quatre vignettes cliniques illustrent comment la capacité négative peut être défaillante chez certains sujets, notamment dans son articulation avec la temporalité, les rendant particulièrement intolérants à la souffrance ordinaire dont parlait Freud et entravés dans l'exploration de leur vie intérieure. Suivant Press, il est par ailleurs proposé que la sollicitation de la capacité négative puisse réactiver chez un individu la crainte de l'effondrement, et avoir pour effet, dans le travail thérapeutique, de mettre à mal la capacité négative du thérapeute. Ce dernier sera amené à devoir tolérer le fait d'occuper transférentiellement auprès de son patient la place de l'objet défaillant, pour permettre que s'actualise le négatif du non-advenu (Press). Il est enfin question de ce qui, dans la formation du psychothérapeute ainsi que dans la culture, peut favoriser ou compromettre le développement de sa capacité négative.
Ce document est protégé par la loi sur le droit d'auteur. L'utilisation des services d'Érudit (y compris la reproduction) est assujettie à sa politique d'utilisation que vous pouvez consulter en ligne.

https://apropos.erudit.org/fr/usagers/politique-dutilisation/ 


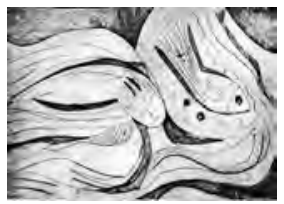

\title{
Transmission de la capacité négative: de la formation à la rencontre clinique
}

\author{
Réal Laperrière
}

Résumé: Inspiré par le poète anglais John Keats, W. R. Bion considérait la capacité négative comme la qualité principale du psychanalyste, et il en faisait aussi pour le patient l'un des buts du traitement analytique. Cette capacité négative peut être mise en lien avec des concepts élaborés par différents auteurs, dont Freud (le masochisme érogène), Rosenberg (le masochisme gardien de la vie), De M'Uzan (l'inquiétude permanente), Press (la construction d'une position passive), Green (la passivité et la passivation), Ribas (la passivité de vie) et Phillips (être un embarras, être perdu, être impuissant). Quatre vignettes cliniques illustrent comment la capacité négative peut être défaillante chez certains sujets, notamment dans son articulation avec la temporalité, les rendant particulièrement intolérants à la souffrance ordinaire dont parlait Freud et entravés dans l'exploration de leur vie intérieure. Suivant Press, il est par ailleurs proposé que la sollicitation de la capacité négative puisse réactiver chez un individu la crainte de l'effondrement, et avoir pour effet, dans le travail thérapeutique, de mettre à mal la capacité négative du thérapeute. Ce dernier sera amené à devoir tolérer le fait d'occuper transférentiellement auprès de son patient la place de l'objet défaillant, pour permettre que s'actualise le négatif du non-advenu (Press). Il est enfin question de ce qui, dans la formation du psychothérapeute ainsi que dans la culture, peut favoriser ou compromettre le développement de sa capacité négative.

Mots clés: capacité négative; temporalité; crainte de l'effondrement; négatif.

\begin{abstract}
Inspired by the English poet John Keats, W. R. Bion saw negative capability as the most important quality a psychanalyst should have, and, for the patient, as one of the main objectives when undergoing therapy. This negative capability can be linked to the concepts formulated by different authors, including Freud (erogenous masochism), Rosenberg (masochism as gardian of life), De M'Uzan (permanent concern), Press (the building of a passive position), Green (passivity and passivation), Ribas (life passivity), as well as Phillips (being an embarrassment, being lost, or being helpless). Four clinical case vignettes show how negative capability can be lacking for some subjects, notably in its articulation with temporality, making them particularly intolerant to ordinary suffering, as described by Freud, and impeded in the exploration of their internal life. According to Press, it is suggested that soliciting negative capability can reactivate an individual's fear of breakdown and can, as a result—in the process of therapy — cripple the therapist's negative capability. The latter will then have to accept to take the place-by
\end{abstract}


transfer-of the defective object in the patient's mind, so that the null and void negative ("le négatif du non-advenu», Press) is actualized. Finally, we address the factors that—-therapist's education or culture-can foster or cripple the building of their negative capability.

Key Words: negative capability; temporality; fear of breakdown; negative.

Denser à la transmission de la psychanalyse, c'est bien sûr s'interroger sur les trajets par lesquels celle-ci s'est rendue à nous, revoir les personnages qui l'ont incarnée pour soi, et tenter de saisir certains effets des empreintes inconscientes laissées par ce parcours sur notre pratique quotidienne, nos idéaux, notre vision de l'humain.

Un regard rétrospectif sur mes nombreuses années d'exercice comme clinicien et superviseur, ainsi que sur ce que j'ai pu en écrire au fil des ans, m’a fait découvrir comment - à mon insu le plus souvent - j'ai toujours tenté, avec plus ou moins de succès, mais sans jamais cesser de m'y accrocher, de tenir un même fil. J'ai un jour appris que ce fil, empreinte laissée par ma formation et ses identifications, avait un nom, la capacité négative, et que c'est à Wilfred R. Bion que l'on devait son inscription dans le vocabulaire de la psychanalyse.

La clinique psychanalytique d'aujourd'hui doit beaucoup à Bion, ce contemporain de Winnicott dont la figure ne me semble pas occuper de nos jours une place équivalente à ce dernier, surtout auprès de mes jeunes collègues. Pourtant, les concepts qu'il a élaborés dans une œuvre il est vrai difficile font maintenant partie des outils de pensée de tout clinicien d'approche psychanalytique. Et certains des plus éminents auteurs contemporains, dont André Green et après lui Antonino Ferro et Thomas H. Ogden, s'en sont directement inspirés pour construire leur propre théorisation.

Dans l'élaboration de son concept de capacité négative, Bion a été inspiré au départ par une lettre du poète anglais John Keats, écrite en 1817, dont voici un extrait:

Plusieurs choses s'emboitèrent dans mon esprit et, à l'instant, je fus frappé par la qualité qui contribue à former un homme accompli, particulièrement en littérature, et que Shakespeare posséda si grandement - je veux parler de la capacité négative, lorsqu'un homme est capable d'être dans l'incertitude, les mystères, les doutes sans courir avec irritation après le fait et la raison. (cité dans Phillips, 2009, p. 7) 
Dans son livre L'attention et l'interprétation (1970), Bion fait de cette capacité négative la qualité principale du thérapeute. Jacqueline PoulainColombier rappelle qu'il la considère comme un «principe d'incertitude» et que, pour lui,

La capacité négative pour le psychanalyste en séance, c’est résister à la tentation de s'accrocher à ce qu'il sait, supporter d'attendre, tout en faisant face à l'épouvante que le travail de curiosité peut provoquer en lui, payer le prix, et pas une fois, à chaque fois. (2014, p. 116).

Bion avait découvert déjà l'importance de la capacité de la mère à tolérer les identifications projectives du petit enfant avant de pouvoir les transformer. Il en conclut donc que l'analyste, lui aussi, doit d'abord se montrer capable de patience (Grotstein, cité dans De Mijolla, 2002). Il doit tolérer la frustration, soutenu par la conviction qu'il sera ainsi possible de trouver éventuellement un sens et de comprendre. Nous nous demanderons plus loin sur quoi peut bien s'appuyer cette conviction, surtout chez le thérapeute qui en est à ses débuts. Cela dit, pour Bion, le thérapeute ne doit

pas tant posséder des connaissances, mais plutôt apprendre à connaitre quelque chose... procédure bien difficile et, peut-être même, contraire à la nature de notre appareil mental, qui semble chercher en permanence la certitude qui guiderait l'action, plutôt que l'exploration des possibles (Casanova Sa, 2013, p. 44).

La capacité négative est donc une capacité en creux, qui exige, comme l'écrit Claudio Neri, de «ne pas donner forme à ce qui est en train d'évoluer et qui pourra prendre forme dans le champ analytique» (2009, p. 51). On rejoint ici l'idée de la saturation ou de la non-saturation de l'écoute et de l'intervention, chère à Antonino Ferro.

Je reviendrai plus loin sur ce qui peut favoriser le développement de la capacité négative du thérapeute, et aussi sur ce qui peut la mettre à mal, dans la rencontre avec le patient, mais aussi dans sa formation et dans la culture. Mais d'abord, si Bion a surtout parlé de la capacité négative du thérapeute, il en a aussi fait un des buts d'une psychanalyse ou d'une psychothérapie. Pour lui, c'est l'intolérance aux espaces vides qui limite la quantité d'espace psychique disponible pour contenir et élaborer, rendant certains individus si intolérants à la frustration (Grotstein, 2016). 
D'autres auteurs, me semble-t-il, ont repris dans des perspectives métapsychologiques différentes cette même idée concernant le but du traitement analytique. Pour Michel de M'Uzan par exemple, le véritable objectif d'une analyse ne serait pas tant d'en arriver à un réaménagement des forces pulsionnelles chez le patient, mais plutôt à ce qu'il parvienne à l'«inquiétude permanente», c'est-à-dire «le point où doit se découvrir, comme donnée fondamentale, le caractère incertain et aléatoire de l'être» $(2015$, p. 8). Le patient est donc convié à vivre dans la rencontre analytique un véritable ébranlement identitaire, où les deux inconscients vont créer un organisme nouveau, qu'il nomme la «chimère». L'inquiétude permanente, une fois l'analyse terminée, va permettre chez le patient l'auto-analyse.

Pour Jacques Press (2010), un des effets transformationnels majeurs d'une analyse est la construction chez le patient d'une position passive, idée qui m’apparait également très proche de la capacité négative. Se référant non pas à Bion, mais à Winnicott, Press propose que c'est cette position passive qui permet au patient d'en venir à tolérer, puis à transformer ce que Winnicott appelait l'«informe», c'est-à-dire non pas le chaos, mais ce qui est en attente de forme et est source de toutes les potentialités ultérieures. Il faut par ailleurs que l'analyste remplisse sa fonction de façon suffisamment bonne pour permettre à ces potentialités d'advenir. C'est ainsi que chez certains patients l'analyse va permettre que s'actualise dans la cure ce que Press (2015) appelle le «négatif du non-advenu », à savoir ce qui de la fonction de l'objet a manqué dans l'histoire du patient, l'expérience qui n'a pu avoir lieu, le travail de transformation qui n'a pas été effectué.

Press rappelle par contre, et en cela il rejoint totalement Bion, que cet informe est difficile à tolérer, tant pour le patient que pour le thérapeute, puisque s'il représente une ouverture aux potentialités, il est aussi désorganisateur pour le moi. C'est pourquoi l'un et l'autre peuvent facilement être amenés à "surthéoriser, à forcer dans une forme connue des pans de réalité» (p. 8), ce qui permet l'illusion de la maîtrise.

Cette "position passive» défendue par Press rejoint la passivité, que Green (1999) différencie de la "passivation». Pour ce dernier, la passivité est le creuset dans lequel l'hallucination négative de la mère permet d'installer un espace propre aux inscriptions psychiques. La passivation pour sa part met l'hallucination négative en échec et plonge le sujet dans la détresse et un éprouvé d'impuissance sans recours.

Ribas (1999), allant dans le même sens, différencie la "passivité de vie», de nature réceptive, de la "passivité de mort», marquée par le désinvestissement, 
le renoncement à l'objet, l'éloignement de celui-ci, un abandon à l'inerte et un vécu de chute que nous retrouverons chez une patiente que j'évoquerai plus loin.

Nous verrons d'ailleurs plus loin ce qui peut sous-tendre chez certains sujets le refus acharné de la position passive, éprouvée essentiellement comme une passivation, refus invalidant toute capacité négative.

Nous pourrions par ailleurs tenter de mettre au travail la notion de capacité négative avec celle de masochisme érogène de Freud (1924) ou encore celle de «masochisme gardien de la vie» de Benno Rosenberg (1991). Disons brièvement que Freud, dans Le problème économique du masochisme, définira le masochisme érogène comme socle de l'investissement de l'espace psychique, permettant à la vie psychique de supporter la tension et les événements psychiques intenses. Emmanuelle Chervet dira que, pour Freud,

La consolidation de ce masochisme érogène est aussi un fruit de la cure: l'enrichissement de l'inconscient secondaire s'étaye sur et nourrit à la fois le masochisme érogène. Une autre butée de la cure sera le refus de la féminité, de la réceptivité (Chervet, 2017, p. 26).

Pour conclure ce survol des concepts apparentés ou redevables à celui proposé et développé par Bion, je ne peux m’empêcher de faire une petite place à l'essai très original d'Adam Phillips (2009) intitulé Trois capacités négatives. Même s'il s'inspire lui aussi du poète Keats, Phillips, curieusement, ne cite à aucun moment Bion, se référant plutôt à Winnicott, Freud, Anna Freud et Lacan. L'auteur étudie trois capacités qu'il définit comme négatives: être un embarras, être perdu et être impuissant. Selon lui, ces capacités sont au fondement de la psyché humaine, et il va jusqu’à affirmer que «La psychanalyse est, entre autres choses, l'histoire de la façon dont nous nous protégeons de notre impuissance, de nos capacités négatives, ainsi que du savoir et de l'expérience que nous en avons» (Phillips, 2009, p. 11).

Pour Phillips, la reconnaissance en soi de ces expériences négatives et surtout la capacité à les tolérer constituent un accomplissement développemental.

Ainsi, être un embarras correspond d'abord à la seule façon par laquelle l'individu peut développer sa confiance en un environnement fiable, qui survit à sa haine. Celui qui ne tolère pas de donner du souci à l'autre a renoncé à l'espoir, et la disposition à déranger et à être dérangé sont essentielles au fait de vivre sa propre vie. 
Ensuite, savoir être perdu va de pair avec la reconnaissance de sa dépendance à l'égard de l'amour de l'objet, de la place qu'on occupe ou non dans son psychisme et de la crainte de perdre cet amour.

Enfin, être impuissant est lié à la capacité de ressentir sa frustration et son besoin des autres. L'impuissance serait donc une condition préalable pour être aidé et éprouver les expériences de satisfaction. «Sans impuissance, écrit Phillips, il n'y a pas de possibilité de satisfaction, et sans possibilité de satisfaction, il n’y a pas de vie, pas de but» (Phillips, 2009, p. 85).

\section{La souffrance ordinaire}

On attribue à Freud la citation suivante: «la psychanalyse vise à transformer la souffrance névrotique en souffrance ordinaire». Un de mes superviseurs, malheureusement disparu, le Docteur Guy Da Silva, faisant toujours les choses à sa manière, reprenait la citation ainsi: "La vie est déjà assez difficile, il n'est pas nécessaire d'en remettre!»

Chez certains de mes patients suivis sur plusieurs années, c'est justement la souffrance ordinaire qui semblait faire problème, en raison d'une intolérance toute particulière aux épreuves inhérentes à la condition humaine. Ce sont eux qui m’ont amené à m’interroger sur la capacité négative.

\section{Première vignette ${ }^{1}$}

La patiente A se présente à sa séance matinale complètement défaite. Elle a pleuré, comme cela lui arrive souvent, une grande partie de la nuit, dans un état de désespoir exempt de toutes pensées. Cet état en est un de «souffrance extrême» (ce sont ses mots), dont elle ne peut rien dire. Peu à peu, quelques mots se déploient, quelques idées s'organisent pour tenter de comprendre cet état. Elle décrit alors l'incertitude de sa vie actuelle: la mise en vente de sa maison, un déménagement éventuel de son bureau professionnel, un voyage, non encore confirmé, avec une amie... Elle se retrouve sans assurance quant à quoi que ce soit. «C'est trop!» dit-elle. «Vous devez donc attendre? » lui dis-je. Elle éclate alors en sanglots: «Attendre! ... attendre! ... c'est intolérable! ... c'est tellement... l'impuissance!»

\section{Deuxième vignette}

La patiente $B$ raconte en séance qu'elle a rêvé la nuit dernière que son ami de cœur la trompait avec une autre. Dès son réveil, elle lui téléphone: «Je sais que tu m’as trompée!» La réaction de surprise de son amoureux, ses questions sur les raisons d'une telle accusation, puis le ton apaisant avec 
lequel il lui dit qu'elle fait erreur ne changent en rien sa conviction: «Alors, si tu ne l'as pas fait, c'est que tu as l'intention de le faire!»

\section{Troisième vignette}

Je reçois le patient $\mathrm{C}$ à sa séance du vendredi, après avoir dû annuler celle du mercredi précédent. Après un moment de réflexion, il dit:

Oui, votre absence de mercredi... ça ne me laissait pas le choix... ça m’a vraiment mis dans l'impuissance... Il faut que j'apprenne à la contenir... Je n'avais pas le choix, fallait que j'attende jusqu'à vendredi... Je pense que ce qui m’a aidé, c'est de savoir que j'allais vous en parler... et aussi que je pourrais être frustré envers vous... que ma colère pourrait... se satisfaire...

Plus loin dans la séance, il évoque les très longues absences de son père lorsqu'il était petit.

J'en viens à lui dire: «Il partait tellement longtemps que vous n'arriviez pas à vous dire: je vais lui en parler quand il sera là... je vais lui dire que je suis fâché.» Le patient pleure en silence.

On peut affirmer, je crois, que ces moments d'analyse avec les patientes A et $B$ illustrent un défaut de capacité négative.

Ainsi, qu'est-ce qui empêche la patiente A de se sentir, pour un temps, impuissante? Pour elle, cette question est irrecevable, impensable. La suite de l'analyse va montrer comment pour elle l'impuissance, même transitoire, convoque un vécu catastrophique. L'incertitude la plonge dans le vide. Attendre est synonyme de désarroi sans appel. Faute de capacité à rêver une issue heureuse, le suspense devient au sens strict invivable: il la renvoie au désarroi radical et au néant de pensée. Il lui faut donc, de façon générale, éviter toute situation d'incertitude, de mystère et de doute. La découverte de sa réalité psychique s'en trouve compromise pour autant.

Lorsque j'essaie d'amener la patiente B à s'interroger sur son rêve, à voir en quoi il pourrait non pas parler de ce que fait son copain ou annoncer ce qu'il va faire, mais plutôt figurer ce qui se passe en elle, rien n'y fait. Elle accueille mes interventions comme une attaque à son narcissisme. Selon elle, je mets en doute les facultés d' «intuition» qui jamais ne l'ont trahie. Je lui dénie ce «don exceptionnel» qui lui sert depuis toujours à prendre des décisions amoureuses, amicales et professionnelles. Il ne servirait à rien de lui rappeler que c'est justement ce qui l'a menée si souvent à l'échec. Elle demeure convaincue, inébranlable. Comme le dirait notre collègue Wilfrid 
Reid (2008), ce qui importe au premier chef pour elle est d'avoir raison, parce qu'elle vit cela comme le garant de son intégrité narcissique. Son intuition est donc pour elle un fait objectif. En d'autres mots, elle ne peut pas être l'indice d'une réalité psychique à découvrir: "c'est comme ça», aussi peu interprétable que le monde extérieur. La réalité intérieure acquiert alors un statut de fait intangible et la résistance au doute, à l'attente, à l'incertitude, dans l'appréciation de celle-ci barre l'accès à toute découverte.

Chez ces deux patientes, l'émergence du sens et l'illusion anticipatrice de la satisfaction sont constamment entravées par la conviction inconsciente qu'il ne peut y avoir d'issue heureuse à leurs vœux.

Avec le patient $\mathrm{C}$, on se situe dans un tout autre registre. L'impuissance éprouvée en réaction à une absence non prévue et imposée par le thérapeute est d'abord reconnue, puis tolérée, et donne lieu à une interrogation sur soi. L'angoisse est nommée, mais peut être contenue par une expérience réussie d'hallucination négative. L'absence permet en effet dans ce cas un espace psychique dans lequel peut s'inscrire une représentation de la satisfaction: «Je vais pouvoir exprimer ma frustration à mon thérapeute». En cours de séance, par le jeu du transfert, une liaison est faite avec le père absent de l'enfance, et la difficulté d'un petit garçon à maintenir en lui son image. Tout ce processus témoigne par ailleurs de l'instauration d'une temporalité fonctionnelle, alors qu'elle était sûrement problématique à certains moments de l'enfance. Or, les troubles de la temporalité m'apparaissent comme un facteur déterminant dans la difficulté à développer la capacité négative. Penchons-nous maintenant sur ce point.

\section{Les troubles de la temporalité}

C'est d'abord dans mon travail auprès des enfants et des adolescents, et plus particulièrement ceux ayant reçu le diagnostic, aujourd'hui fort répandu, de "trouble anxieux", que j'ai pris conscience du rôle de la temporalité, plus exactement des particularités de celle-ci, dans de telles conditions psychiques. Comment en effet ne pas vivre dans un état permanent d'hypervigilance inquiète lorsque, sur la scène inconsciente, les mots «demain », «bientôt», «un jour», "plus tard», etc. s'avèrent équivalents, par une sorte de collapsus du temps? Lorsque toute émergence de pensée, fantasme ou représentation est éprouvée comme devant donner lieu à une réalisation immédiate, et a alors un impact traumatique? Il est fascinant de voir comment ces enfants et adolescents en situation de jeu psychodramatique n'arrivent pas à déployer dans le temps, au moment de la jouer, 
l'histoire élaborée souvent laborieusement en groupe. Tout est alors télescopé (le début de l'histoire, son déroulement, sa fin) et, sans l'intervention d'un thérapeute meneur de jeu, tout serait conclu, ou devrais-je dire évacué, en moins de deux minutes.

Dans son bel ouvrage intitulé Les désordres du temps, Jacques André (2010) soutient que le rapport subjectif au temps n'est pas le même pour tous et que les mots associés aux catégories de passé, de présent et de futur peuvent avoir des significations différentes selon l'histoire de chacun. La temporalité, c'est-à-dire l'inscription de la vie psychique dans le temps et, ajouterais-je, du temps dans la vie psychique, n'est pas, selon André, une donnée immédiate, mais le résultat d'un processus, qu'il ne faut pas confondre par ailleurs avec le développement des fonctions cognitives: les personnes présentant un trouble de la temporalité n’ont pas nécessairement de problème à fonctionner avec le temps social. C'est au niveau de l'éprouvé subjectif de ce temps que se situe la difficulté.

Ainsi par exemple, pour certains, le futur n'existe pas, et la moindre évocation de quelque chose à venir les plonge dans une angoisse liée à un vécu de non-préparation traumatique, comme si tout ne pouvait arriver que de façon soudaine et inattendue, ce qui affecte parfois dramatiquement, comme nous le verrons, l'expérience de continuité d'être (Winnicott).

Chez d'autres, c'est le passé qui n'existe pas. Ils n'ont pas de problèmes de mémoire, dit Jacques André, mais un problème d'histoire, celle-ci ne se déclinant pas au passé mais plutôt à l'impassé, comme nous l'a montré Dominique Scarfone (2014).

Enfin, certains ne semblent pouvoir fonctionner dans la catégorie du présent, ayant toujours l'impression d'être à côté, ailleurs et là en même temps, mais pas vraiment là; dans le hors de soi et le ne pas être là si bien décrits par Ferenczi dans son journal clinique (1932, p. 79-80). Un homme me disait récemment souffrir de tout faire à la course, sans plaisir, comme s'il fallait au plus vite passer à autre chose... mais à quoi, au juste? Il me dit s'être senti très apaisé durant la semaine suivant une rencontre où je lui proposai que son temps ne semblait pas lui appartenir et que, par conséquent, il ne pouvait pas en jouir... Il ramena cette idée à son histoire d'enfant, période pendant laquelle il dut veiller sur une mère endeuillée, suite à la mort tragique de deux de ses enfants.

Pour Jacques André, faire l'expérience de la continuité d'être dans un environnement offrant suffisamment de permanence est préalable à l'acquisition du découpage du temps. 
François Gantheret, dans La nostalgie du présent (2010), va dans le même sens, en s'appuyant pour sa part sur des textes de Freud où celui-ci relie la naissance de la temporalité au rythme présence/absence de la satisfaction; et au texte de Winnicott sur la préoccupation maternelle primaire. Pour Gantheret, chaque défaillance de cette fonction ferait vivre à l'enfant une menace d'annihilation, et la première organisation du moi - et on peut penser de la temporalité - proviendrait du vécu de menaces d'annihilation qui ne se réalisent finalement pas et dont on se remet ainsi à chaque fois.

\section{Quatrième vignette}

Pouvoir se formuler à elle-même, avec suffisamment de conviction, une phrase telle que «Des moments difficiles, il y en aura toujours, mais je sais maintenant qu'ils passent», cela risque de paraitre bien modeste comme résultat de nombreuses années d'analyse avec Madame D. Et pourtant, nous avons dû traverser d'innombrables moments de désespoir, de vécus d'agonie, de somatisations invalidantes et de pressions dans l'urgence sur le cadre et sur l'analyste pour que s'instaure une temporalité offrant des possibilités de profiter du moment présent et d'établir des rapports moins conflictuels avec ses proches.

«Je ne sais pas si je vais m'en sortir»: cette phrase fut sans cesse répétée dès les premiers moments de notre travail ensemble. Madame D. vit alors des accès d'angoisse massive, qui lui font craindre de devenir folle et de commettre des gestes violents. Elle redoute également - et espère secrètement, comme nous le découvrirons - une maladie mortelle en phase terminale, dont ses nombreux malaises physiques seraient les signes annonciateurs.

C'est en analysant son rapport à la cigarette que je suis mis sur la piste de la temporalité. Madame $\mathrm{D}$. se sent très coupable et angoissée de nuire à sa santé, mais découvre que les pauses qu'elle prend dans la journée pour fumer lui permettent de marquer le temps. Aller d'une pause à l'autre instaure une temporalité artificielle la sortant d'un angoissant vécu de suspension dans le vide où temps et mouvement n'existent pas. Peu à peu, ses trois séances hebdomadaires semblent prendre le relais: elle laisse son paquet de cigarettes caché dans ma salle d'attente, et sa consommation se limite pendant un temps à ce qu'elle fume après chaque séance, jusqu'à ce qu'elle cesse complètement.

Pour Madame D., le temps subjectif semble être celui du pensionnat où elle a été placée dès l'âge de cinq ans et pour toutes ses années d'études primaires et secondaires. Un temps suspendu, infini, où l'attente des visites 
de la mère ou des retours à la maison perdait tout sens, puisqu'elle dépassait ses capacités hallucinatoires. À la place de l'anticipation de la satisfaction s'installait en elle le long, éprouvé comme une éternité.

Madame $\mathrm{D}$. ne garde à peu près pas de souvenirs de toutes ses années au pensionnat: des portes immenses se refermant derrière elle, des corridors sans fin, des religieuses qui, dans leurs habits ne dévoilant que le visage, lui semblaient ne pas avoir de corps. Elle découvre par ailleurs au fil des séances ce qu'elle qualifie de moyens de survie. D'abord, la pratique du piano, quotidienne, enfermée dans un cubicule à faire répétitivement ce qu'elle affectionnait alors le plus, des gammes. Temporalité artificielle, marqueuse de temps, mais un temps excluant toute pensée d'attente, de désir, de manque.

Puis, la maladie apparut comme une modalité d'automaintien. Elle se souvient de plusieurs séjours à l'infirmerie, dont un qui aurait duré trois mois, autour de l'âge de sept ans. Elle se revoit couchée et fiévreuse, mais apaisée, simulant le temps arrêté de la mort, comme pour s'en protéger.

Pendant longtemps, le futur n'existe pas pour Madame D. L'attente peut lui faire vivre de véritables moments de désespoir, avec idées suicidaires, puisque sur la scène inconsciente prévaut la conviction que ce qui n'arrive pas maintenant, tout de suite, n'arrivera jamais. De même, l'angoisse peut devenir intolérable dans des situations imprévues ou inhabituelles, qui m’apparaissent à moi comme des irritants propres à la vie ordinaire, mais qui sont toujours vécues comme de véritables gouffres sans fond. Tout est trop long pour elle, comme le long sans fin du pensionnat. De la même façon, mes vacances lui semblent, elles aussi, «trop longues», et la possibilité d'un contact téléphonique pendant celles-ci (que par ailleurs elle n'utilisera jamais) lui est nécessaire au cours des premières années de l'analyse. Nous découvrirons enfin ensemble que sa peur d'avoir une maladie mortelle et d'être devant une fin imminente traduit le désir de retourner à l'infirmerie et d'y retrouver le temps arrêté. Elle décrira pendant plusieurs séances son sentiment de flotter au-dessus du divan, sans angoisse, hors de son corps.

Évidemment, bien d'autres dimensions feront l'objet de notre travail ensemble. Mais la difficulté à instaurer une temporalité permettant l'actualisation de la capacité négative a été déterminante dans cette analyse.

\section{La capacité négative du thérapeute}

Notre capacité négative est toujours plus ou moins défaillante, et Adam Phillips nous montre bien comment il est dans notre nature de ne jamais cesser de vouloir nous protéger de toute circonstance la sollicitant. Nous 
rencontrons toutefois des patients chez qui ce besoin de protection est plus important, au point de poser des difficultés particulières au thérapeute.

Chez ces personnes, la mobilisation émotionnelle est très souvent vécue comme une catastrophe, et les met en situation d'urgence. Je suis en accord avec Jacques Press (2010) lorsqu'il propose que, chez celles-ci, toute situation comportant une certaine dose de passivité réactive ce que Winnicott a appelé la "crainte de l'effondrement». Elles vivent un éprouvé d'effondrement imminent, de catastrophe à venir, alors que ce qu'elles redoutent est une projection dans le futur immédiat de ce qu'elles ont déjà vécu autrefois et qui alors n'a pu être éprouvé, ne laissant qu'une trace en creux. Cette urgence catastrophique les pousse à faire une forte pression sur le thérapeute pour qu'il fasse quelque chose afin de les apaiser. La conviction inconsciente du patient quant à la non-réalisation de son désir et de l'imminence de l'effondrement (tomber à jamais, ne pas pouvoir s'en sortir, etc.) se transmet subrepticement au thérapeute qui, mobilisé alors lui aussi par ce que Reid (2004) nomme le «tout pouvoir de l'affect», devient lui-même convaincu de l'incapacité de son patient à tolérer ce qu'il est en train de vivre; qu'il y a un danger bien réel de régression dramatique ou même de passage à l'acte fatal, et qu'il y a urgence à interpréter, conseiller et même agir. Reid écrit ainsi que «Le trauma psychique, peut-on penser, est aussi contagieux qu'une infection virale, de sorte que le fonctionnement psychique traumatique de l'analyste» - qui consiste à évacuer la surcharge affective plutôt que de procéder à une élaboration contre-transférentielle «devient un excellent indicateur du fonctionnement traumatique de l'analysant» (2004, p. 35).

On sait pourtant que la réaction dans l'urgence du thérapeute, très souvent, ne fait que confirmer la conviction négative du patient, alors que sa capacité à recevoir et à tolérer la charge émotionnelle ainsi qu'à amener le patient à s'interroger sur celle-ci a plus de chances d'apporter un apaisement authentique.

D'autre part, ces patients ont besoin de pouvoir utiliser les failles du thérapeute, ses manquements à la technique, au tact et parfois même à l'éthique - à condition que ces manquements ne conduisent pas à une rupture du traitement - pour faire ou refaire dans le transfert l'expérience d'un objet défaillant et d'un environnement où ce qui devait se produire ne s'est pas produit. Les patients doivent pouvoir refaire cette expérience, mais en pouvant maintenant, avec l'aide du thérapeute, l'inscrire psychiquement dans le temps. On rejoint ici le négatif du non-advenu de Jacques Press. 
Thomas Ogden (2015) propose en outre que l'effondrement appréhendé par ces patients est en fait celui de la relation mère-nourrisson, qui aurait laissé l'enfant seul, à la limite de la non-existence, et empêché l'expérience précoce de l'omnipotence, nécessaire à l'établissement d'une fonction hallucinatoire opérante permettant l'anticipation du positif. Dans la relation thérapeutique, ces patients vont en venir à fortement reprocher au thérapeute, de façon plus ou moins manifeste, de ne pas être là pour eux, alors que celui-ci se sent très engagé dans la thérapie. Ils vont œuvrer à négativer la présence de l'objet qui contient, et à positiver un objet abandonnant. C'est ainsi par exemple que le pas là d'une absence du thérapeute va complètement effacer le là de toutes ses présences régulières et prévisibles... du moins pour un temps. Celui-ci devra tolérer d'occuper cette place d'objet absent et manquant, tout en étant présent au vécu d'isolement du patient, et ce, afin de l'aider à l'éprouver et à le comprendre.

Enfin, la confrontation par le patient à ce qui n’a pas été vécu, éprouvé, représenté, exige de l'analyste qu'il mobilise sa capacité à rêver avec et pour lui ce qui ne se présente pas dans le matériel de la séance, ou ne s'y présente qu'en creux. Seule la tolérance au fait de ne pas comprendre peut alors permettre l'exploration des possibles (Casanova Sa, 2013).

\section{Apprendre la capacité négative?}

Si la capacité négative est la qualité première du psychothérapeute ou du psychanalyste, il devient troublant de penser que ce sur quoi repose sa compétence ne s'apprend pas et échappe au cursus académique. Comment s'acquiert-elle alors, se développe-t-elle et surtout se maintient-elle dans son travail clinique? Certains en sont certainement mieux pourvus au départ, en raison de leur histoire et de leurs identifications. Mais l'analyse personnelle en favorise certainement le développement. La temporalité particulière qui s'installe dans un cadre analytique à haute fréquence (Scarfone, 2015), c'està-dire ayant une cadence élevée de séances et utilisant le dispositif divan/ fauteuil, avec son dosage suffisant de frustration permettant la prise de contact avec l'angoisse et l'ouverture aux manifestations de l'inconscient et aux résistances à celles-ci, constitue ce qui tout à la fois développe la capacité négative et la nécessite.

Pour le thérapeute en début de carrière, le défi est de taille: l'analyse personnelle souvent n'est pas encore commencée, parfois parce que le besoin ne s'en est pas encore fait sentir. Le recours à la méthode analytique avec des patients repose alors sur un véritable acte de foi, l'expérience de son 
efficacité n'en ayant pas encore été faite, ni avec des patients, ni pour soimême. Le rapport au superviseur et l'identification à la capacité négative de celui-ci deviennent alors indispensables, en autant que ce dernier en fasse lui-même preuve dans son travail avec le supervisé, et qu'il ne se réfugie pas dans une position visant à faire la démonstration de sa maîtrise et de son savoir. J'ai toujours pensé que l'essentiel de la tâche du superviseur auprès d'un thérapeute en début de pratique consiste à aider celui-ci à trouver ce qu'il a en lui pour faire ce travail. «Apprendre à faire le mieux possible avec ce que l'on a», comme le disait notre collègue Jean Bossé.

Il me semble par ailleurs que la formation actuelle donnée à ceux qui se destinent à la pratique de la psychothérapie soit peu favorable au développement de la capacité négative. Je pense surtout ici à la formation des psychologues, qui est celle que je connais. Il m'apparait que les exigences de savoir, de maîtrise et de contrôle n'ont cessé d'augmenter avec les années et se sont imposées comme garantes de la compétence. De même, dans un souci légitime d'assurer la place de la psychologie dans le monde professionnel, l'Ordre des psychologues a pris le relais des universités en défendant l'image du psychologue comme expert, c'est-à-dire possédant un savoir sur son patient, dans une promotion du savoir plutôt que de la capacité à apprendre grâce au patient et surtout avec le patient. Mais de quelle expertise peut se réclamer le psychothérapeute analytique; «expert de l'inconscient»? La formule a de quoi faire sourire...

Mon expérience de superviseur m'a souvent fait rencontrer de jeunes psychologues particulièrement brillants ayant appris à penser par euxmêmes, et pour certains, pouvant compter sur les connaissances chèrement acquises à l'université pour entreprendre des psychothérapies. Or, souvent avec surprise, et parfois pour la première fois dans leur parcours de formation, ils se montrent très angoissés par la rencontre avec leurs patients, vivant alors un douloureux sentiment d'incompétence qu'ils n'arrivent pas à comprendre... et qui les amène en supervision. Ils vont alors devoir tolérer de laisser de côté ce qu'ils ont appris: le devoir de performer, de comprendre, d'expliquer, d'analyser... pour en développer le négatif. Or, comme l'écrit Adam Phillips, la culture contemporaine - et, pourrions-nous dire, notre monde professionnel - fait l'idéalisation «d'un individu plein de ressources, d'une personne qui sait ce qu'elle veut et comment l'obtenir» (2009, p. 115).

Il ne faudrait pas croire pour autant que le thérapeute d'expérience peut pour sa part compter sur une capacité négative éprouvée et acquise. De la même façon que le psychanalyste demeure toujours confronté à ses propres 
résistances à l'inconscient, sa capacité négative dans son travail avec un patient est toujours menacée. Il s'agit d'une position à tenir, bien davantage que d'une réalisation.

Je terminerai par des mots du regretté Réjean Ducharme, tirés de son roman Le nez qui voque (1967): «Comme tous les êtres humains fuient, ceux qui sont à l'avant-garde sont ceux qui fuient le plus vite.»

\section{Réal Laperrière laperrierereal@videotron.ca}

\section{Notes}

1. La première et la deuxième vignettes ont été publiées dans Laperrière, 2016.

\section{Références}

André, J. (2010). Les désordres du temps. Paris: Presses universitaires de France.

Bion, W. R. (1970). L'attention et l'interprétation. Une approche scientifique de la compréhension intuitive en psychanalyse et dans les groupes. Paris: Payot.

Casanova Sa, M. T. (2013). Clinique et éducation: dialogues avec notre humaine condition. Cahiers de psychologie clinique, 2 (41), 37-48.

Chervet, E. (2017). Patient, et interprète. Le domaine intermédiaire. Revue française de psychanalyse, 81 (5), 1301-1365.

De Mijolla, A. (dir.) (2002). Dictionnaire international de la psychanalyse. Paris: Calman-Lévy.

Ducharme, R. (1967). Le nez qui voque. Paris: Gallimard.

Ferenczi, S. (1985). Journal clinique. Janvier-octobre 1932. Paris: Payot.

Freud, S. (1924). Le problème économique du masochisme. Dans S. Freud, Du masochisme. Paris: Payot.

Gantheret, F. (2010). La nostalgie du présent. Psychanalyse et écriture. Paris: Éditions de l'Olivier.

Green, A. (1999). Passivité et passivation: jouissance et détresse. Revue française de psychanalyse, 63 (5), 1587-1600.

Grotstein, J. S. (2002). Capacité négative. Dans A. De Mijolla (dir.), Dictionnaire international de la psychanalyse. Paris: Calmann-Lévy.

Grotstein, J. S. (2016). Un rayon d'intense obscurité. Ce que Wilfred R. Bion a légué à la psychanalyse. Paris: Les éditions d'Ithaque.

Laperrière, R. (2016). Capacité négative et capacité de rêverie. Dans L. Danon-Boileau et J.-Y. Tamet (dir.), Des psychanalystes en séance. Glossaire clinique de psychanalyse contemporaine (p. 130-133). Paris: Gallimard.

M'Uzan, M. de (2015) L'inquiétude permanente. Paris: Gallimard.

Neri, C. (2009). La capacité négative du psychothérapeute de groupe. Dans R. Kaës et P. Laurent (dir.), Le processus thérapeutique dans les groupes (p. 51-66). Paris: Dunod.

Ogden, T. H. (2015). La crainte de l'effondrement et la vie non-vécue. L'année psychanalytique internationale, 2015 (1), 19-40.

Phillips, A. (2009). Trois capacités négatives. Paris: Éditions de l'Olivier.

Poulain-Colombier, J. (2014). De la «capacité négative» comme «principe d'incertitude». Le Coq-Héron, 1 (216), 114-116. 
Press, J. (2010). La construction du sens. Paris: Presses universitaires de France.

Press, J. (2015). Enjeux épistémologiques et cliniques de la recherche en psychosomatique. Manuscrit inédit.

Reid, W. (2004). Le contre-transfert, la symbolisation et le don d'absence. Filigrane, 13 (2), 34-47.

Reid, W. (2008). Un nouveau regard sur la pulsion. Le trauma et la méthode analytique. Première partie: une théorie de la psyché. Filigrane, 17 (1), 68-94.

Reid, W. (2008). Un nouveau regard sur la pulsion. Le trauma et la méthode analytique. Deuxième partie: une théorie de la méthode. Filigrane, 17 (2), 70-98.

Ribas, D. (1999). Passivité de vie, passivité de mort. Revue française de psychanalyse, 63 (5), 1647-1650.

Rosenberg, B. (1991). Masochisme mortifere et masochisme gardien de la vie. Paris: Presses universitaires de France.

Scarfone, D. (2014). L'impassé, actualité de l'inconscient. Bulletin de la Société psychanalytique de Paris, 2014 (1), 207-231.

Scarfone, D. (2015). La formation de l'esprit psychanalytique. Bulletin de la Société psychanalytique de Montréal, 27 (1), 28-33. 